\title{
A Comparative Study of Control Methods for a Robotic Manipulator with Six DOF in Simulation
}

\author{
Georgia P. Smyrnaiou*, Michail Papoutsidakis and Dimitrios Tseles \\ Piraeus University of Applied Sciences, Department of Automation Engineering, 12244, P. Ralli \& Thivon 250, Athens, Greece
}

\begin{abstract}
In this paper a comparative study of the classical control methods for the testing of a mathematical model, which controls six actuators of a six degrees of freedom robotic arm with a single controller, is illustrated, aiming to the constructive simplification of the system. In more detail, a mathematical model of the system is designed which simulates all mechanical parts, including 5-way directional pneumatic valve, the pneumatic actuators/pistons and the mathematical model of the controller. The purpose of the above is the tuning of a Single Input, Multiple Output (SIMO) controller which will direct the motion of the six pneumatic pistons. The thorough analysis of the implementation of the pneumatic system in Matlab/Simulink environment is followed by experimentation and results using Proportional (P), Proportional-Integral (PI), Proportional-Derivative (PD) and Proportional-IntegralDerivative (PID) controllers. The simulation results show the advantages of the above classical control methods on the robotic human arm which imitating human motion and made by a well-known company in the field of pneumatic automation.
\end{abstract}

\section{Introduction}

In modern industry pneumatic systems are used with an increased rate. A pneumatic system uses compressed air as a mean to transfer the motion, and have as a final result the movement of a piston or in rare cases the rotation of a motor. Pneumatic Systems find application in cases where small forces should be applied and great velocity is needed. This is the main reason they are widely used in the industry. Pneumatic systems also store and transfer energy with ease and can produce low cost, linear movement with relatively high speed, easily adjustable [1].

Cylinders are considered the basic elements of motions in Pneumatic Systems, which convert the pneumatic force into a linear motion. With the pneumatic cylinders achieve the reciprocating motion by means of compressed air.

Pneumatic cylinders consist of a chamber and ports from where the air enters and exeunt, performing, that way, the linear movement. The piston's chamber is tight to avoid any leakage and is highly durable so that it can endure the high pressure [2].

There are three distinguished categories for the pneumatic cylinders:

- The single-acting cylinders

- The double-acting cylinders

- The Tandem cylinder.

For the correct function of the pneumatic actuators a controller is required. The valves are devices that help regulate the starting or stopping of the piston, and also assist in determining the compressed air flow direction. The valve controls the motion of the rod and the valve respectively is tuned by a classic controller [3]. Valves are distinguished in the following categories:

- Directional valves.

○ Signal valves.
- Pressure control valves.

○ Return / check valves [2].

\section{Control appliance in Simulation environment}

\subsection{Non-linear mathematical model}

A pneumatic system consists of a cylinder, a valve, exhaust and supply tubes, position sensors and pressure sensors. The load is the rest of the mechanical parts that are connected to the pneumatic piston. A non-linear pneumatic system is illustrated in the following Figure.

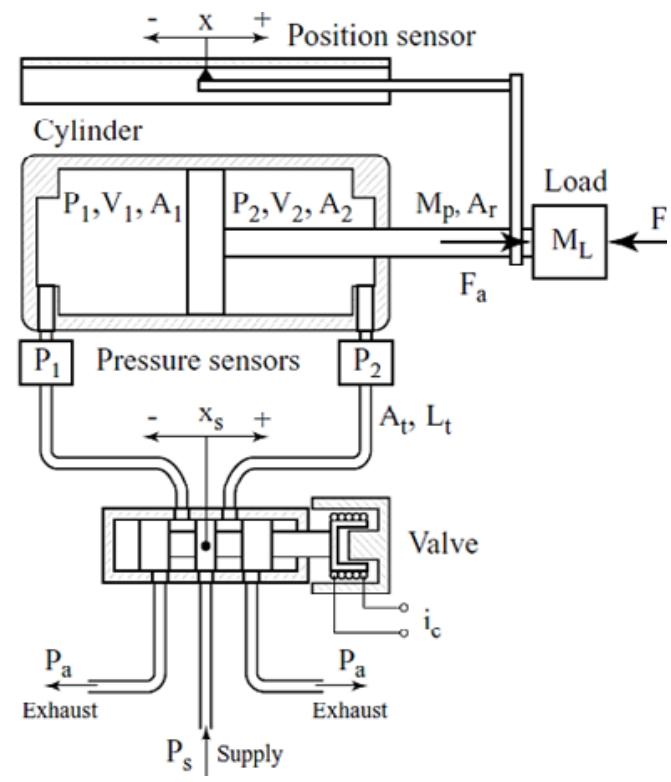

Fig. 1: Standard pneumatic cylinder-valve model

* Corresponding author: giouli.smyrnaiou@gmail.com

(C) The Authors, published by EDP Sciences. This is an open access article distributed under the terms of the Creative Commons Attribution License 4.0 (http://creativecommons.org/licenses/by/4.0/). 
The equation of motion for the piston-rod-load assembly can be expressed as:

$$
\begin{aligned}
& \left(\mathrm{M}_{\mathrm{L}}+\mathrm{Mp}\right) \cdot \ddot{\mathrm{x}}+\mathrm{B} \cdot \dot{\mathrm{x}}+\mathrm{F}_{\mathrm{f}}+\mathrm{F}_{\mathrm{L}} \\
& =\mathrm{P} 1 \cdot \mathrm{A} 1-\mathrm{P} 2 \cdot \mathrm{A} 2-\mathrm{P}_{\mathrm{I}} \cdot \mathrm{A}_{\mathrm{r}}
\end{aligned}
$$

Where $M_{L}$ is the external mass of the load, $M p$ is the piston and rod assembly mass, $\mathrm{x}$ is the piston position, $\dot{\mathrm{x}}$ is the velocity of the piston, $\ddot{x}$ is the acceleration of the piston, $B$ is the friction coefficient, $F_{f}$ is the friction force (Coulomb), $\mathrm{F}_{\mathrm{L}}$ is the external force, $\mathrm{P} 1$ and $\mathrm{P} 2$ are the absolute values of the air pressure of the actuator's chambers, $\mathrm{P}_{\mathrm{a}}$ is the absolute value of the environment pressure, $\mathrm{A} 1$ and $\mathrm{A} 2$ are the piston effective areas, $\mathrm{A}_{\mathrm{r}}$ is the rod cross sectional area.

The above equation represents the force of the actuator generated by different pressures acting on the opposite sides of the piston, in order to control the actuator's output.

The time derivative for the pressure in the pneumatic cylinder chambers can be expressed as:

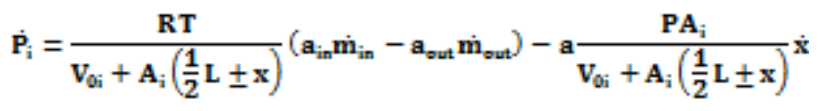

Where $\mathrm{P}$ is the piston's pressure, $\mathrm{R}$ is the ideal gas constant, $\mathrm{T}$ is the temperature, $\mathrm{V}_{\mathrm{oi}}$ is the inactive volume at the end of stroke and admission ports, $A_{i}$ is the piston effective area, $\mathrm{L}$ is the piston stroke, $\mathrm{x}$ is the piston position, $m_{\text {in }}$ and $m_{\text {out }}$ are the mass flows entering and leaving the chamber, and $a_{\text {in }}, a_{\text {out }}$ taking values between 1 and $\mathrm{k}$, depending on the actual heat transfer during the process.

The first term of the above equation represents the effect of the pressure of the air flow into or out of the cylinder chamber, while the second term describes the effect of the motion of the piston.

The pressure drop across the valve orifice is usually large, and the flow has to be treated as compressible and turbulent. If the upstream to downstream pressure ratio is larger than a critical value $\mathrm{P}_{\mathrm{cr}}$, the flow will attain sonic velocity (choked flow) and will depend linearly on the upstream pressure. If the pressure ratio is smaller than $\mathrm{P}_{\mathrm{cr}}$ the mass flow depends nonlinearly on both pressures. The standard equation for the mass flow through an orifice of area $A_{\mathrm{u}}$ is:

$$
\dot{m}_{u}= \begin{cases}C_{f} A_{u} C 1 \frac{P_{u}}{\sqrt{T}} & \text { if } \frac{P_{d}}{P_{u}} \leq P_{c r} \\ C_{f} A_{u} C 2 \frac{P_{u}}{\sqrt{T}}\left(\frac{P_{d}}{P_{u}}\right)^{\frac{1}{k}} \sqrt{1-\left(\frac{P_{d}}{P_{u}}\right)^{\frac{(k-1)}{k}}} & \text { if } \frac{P_{d}}{P_{u}}>P_{e r}\end{cases}
$$

Where $\dot{m}_{u}$ is the mass flow through valve orifice, $C_{f}$ is the supply rate, $P_{u}$ is the value of the upstream pressure and $P_{d}$ is the value of the downstream pressure. $\mathrm{C} 1$ and $\mathrm{C} 2$ are constants of a given fluid, in this case the air:

$$
\mathbf{C} 1=\sqrt{\frac{k}{R}\left(\frac{2}{k+1}\right)^{\frac{k+1}{k-1}}}, C 2=\sqrt{\frac{2 k}{R(k-1)}} \text { and } P_{e r}=\left(\frac{2}{k+1}\right)^{\frac{k}{k-1}}
$$

Where $\mathrm{k}$ is the constant of air value [4].

\subsection{Simulation of the Pneumatic System with six-pistons and Proportional controller (P)}

The pneumatics' system simulation model with six parallel connected pistons with analog control is illustrated in the figure below. 


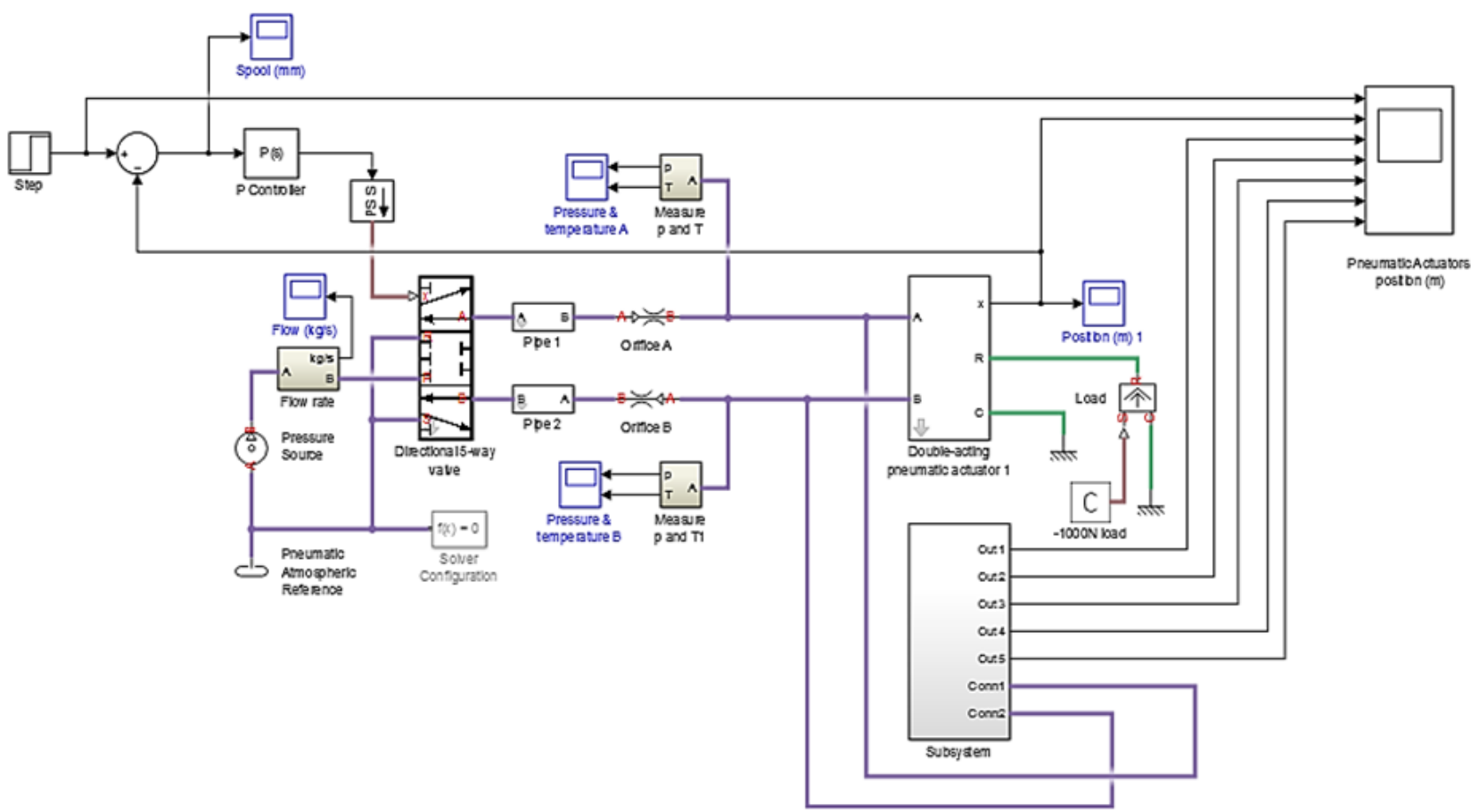

Fig. 2: Simulation model of the pneumatic system with six pistons and $\mathrm{P}$ controller

This model represents an integrated pneumatic system with six controllable actuators/ pistons. The components are described below:

\section{A directional control valve $5 / 3$}

This is a five-way valve and three-position ( $5 / 3$ valve) which is used to control the compressed air's flow into and out the pistons, having as an output the return of the rods.

As shown in Fig.2, the supplied pressure is inserted in port $\mathrm{P}$ and the pneumatic actuators are connected to ports $\mathrm{A}$ and $\mathrm{B} . \mathrm{R}$ and $\mathrm{S}$ are return paths of the $\mathrm{A}$ and $\mathrm{B}$ ports respectively.

\section{Double-acting Cylinders}

The Pneumatic System uses six double-acting cylinders with a single rod that forces the compressed air to move the rod in both directions.

\section{Flow Rate}

As shown from Fig. 2, the block diagram of the flow rate of the pneumatic system is connected with the directional valve to drive the compressed air to the actuators ports so that they can move accordingly. The Flow rate block diagram contains a subsystem which consists:

- Mass \& Heat Flow Sensor, which is a device that converts the mass flow rate and heat flow rate between the two pneumatic nodes into physical measurement signals $\mathrm{G}$ and $\mathrm{Q}$. The positive direction of the sensor is from the port A to port B.

o PS-Simulink Converter which converts the input Physical Signal to a unit less Simulink output signal in this case $\mathrm{Kg} / \mathrm{s}$.

\section{Pressure Source}

This is an ideal air compressor which maintains a fixed pressure differential, irrespectively the flow rate. The compressor does not add extra heat to the system. A positive pressure difference results in the pressure in port $\mathrm{B}$ to be greater than the pressure in the port $\mathrm{A}$. The compressor differential pressure is 6 bar.

\section{Subsystem block and Time scope block}

For the pneumatic system Simulation, the Simulink library was used. Time scope block is used and the six actuators are connected and also the input step. A Subsystem block is created which includes the five pneumatic pistons and it's connected with the first one (Master-slaves) as illustrated in the following figure.

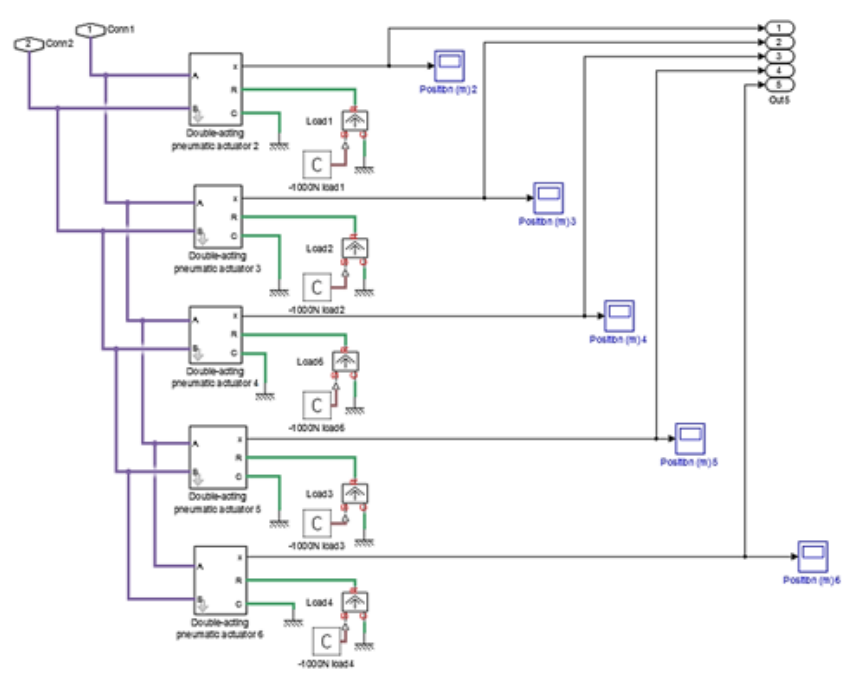

Fig. 3: Subsystem block 
The Step input is set to start from 0 sec. Step's range value starts from 0 to 1 .

As final value in the stoke parameter of the pistons is set $0.05 \mathrm{~m}$ height and the stroke length is set in $0.1 \mathrm{~m}$.

From the Simulink library a PID controller is selected (Proportional-Integral-Derivative). The $\mathrm{P}$ value is set to $\mathrm{K}_{\mathrm{p}}=120$ and the rest of the parameters are eliminated (0) to achieve Proportional control. Simulation time is set to $\mathrm{T}=0.5 \mathrm{sec}$, air supply pressure is set to $6 \mathrm{bar}$, $\left[6 \cdot 10^{5}\right.$ Pascal $]$.

The systems response and the position of the six actuators with Proportional control are shown in the following figure.

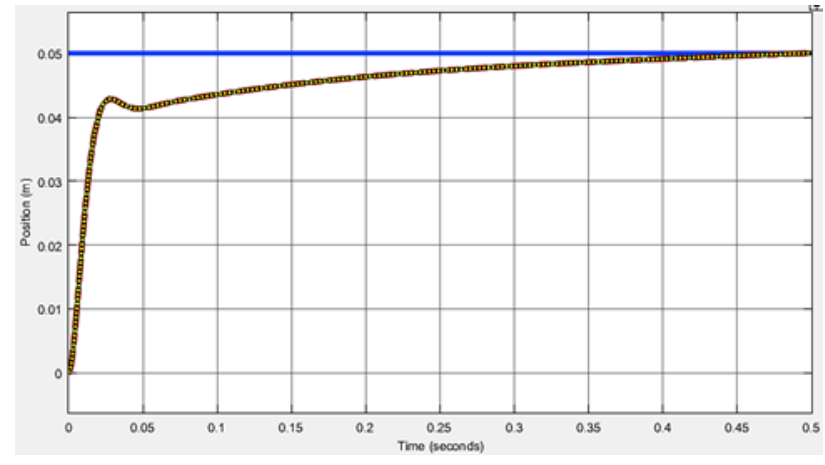

Fig. 4: P controller's Step Response

The fig. 4 results for $\mathrm{K}_{\mathrm{p}}=120$, where the pistons have settled in the Set value. For lower $\mathrm{K}_{\mathrm{p}}$ values a steady state error occurs in the systems response with values on the position of the pistons less than $0.05 \mathrm{~m}$. For greater $\mathrm{K}_{\mathrm{p}}$ values the steady state error is lifted. At this point it should be mentioned, that the steady state error is not erased completely, but instead it is getting smaller.

\subsection{Simulation of the Pneumatic System with six-pistons and Proportional-Integral controller} (PI)

The PID controller is tuned (Proportional-IntegralDerivative) to $\mathrm{K}_{\mathrm{p}}=120$ and $\mathrm{K}_{\mathrm{I}}=5$ to achieve Proportional-integral control. The following figure illustrated the pneumatic system response.

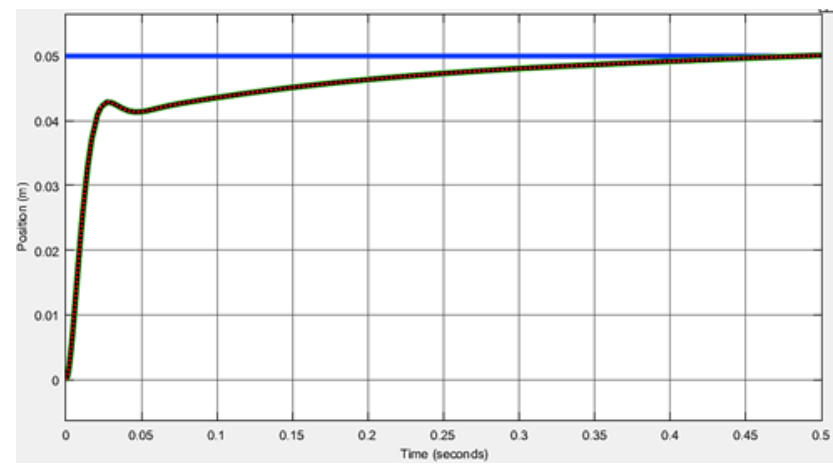

Fig. 5: PI controller's Step Response

The above figure's results for $\mathrm{K}_{\mathrm{p}}=120$ and $\mathrm{K}_{\mathrm{I}}=5$, where the pistons have settled in the Set value.
For increased the $\mathrm{K}_{\mathrm{I}}$ gain, a steady state error appears in the system with values of the position of the pistons greater than $0.05 \mathrm{~m}$.

\subsection{Simulation of the Pneumatic System with six-pistons controller (PD) and Proportional-Derivative}

The PID controller is tuned (Proportional-IntegralDerivative) to $K_{p}=120$ and $K_{D}=0.6$ to achieve Proportional-Derivative control in the pneumatic system. The following figure illustrated the pneumatic system response.

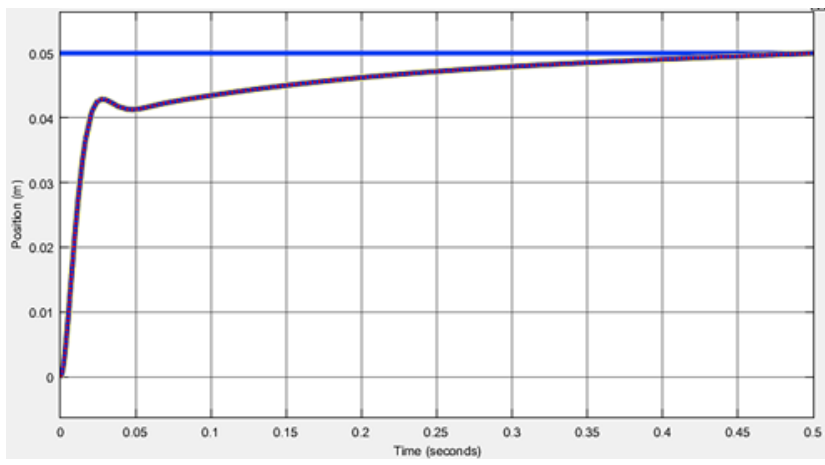

Fig. 6: PD controller's Step Response

The above figure, results for $\mathrm{K}_{\mathrm{D}}=0.6$ and $\mathrm{K}_{\mathrm{p}}=120$ and filter coefficient $\mathrm{N}=100$, where the pistons have settled in the Set value.

Increasing the $K_{D}$ coefficient a steady state error occurs with values on the position of the pistons less than 0.05 m.

\subsection{Simulation of the Pneumatic System with six-pistons and Proportional-Integral-Derivative controller (PID)}

The PID controller is tuned (Proportional-IntegralDerivative) to $\mathrm{K}_{\mathrm{p}}=120, \mathrm{~K}_{\mathrm{I}}=5$ and $\mathrm{K}_{\mathrm{D}}=0.6$ to achieve Proportional-Integral-Derivative control in the pneumatic system.

The system response and the position of the six actuators with Proportional-Integral-Derivative control is shown in the following figure.

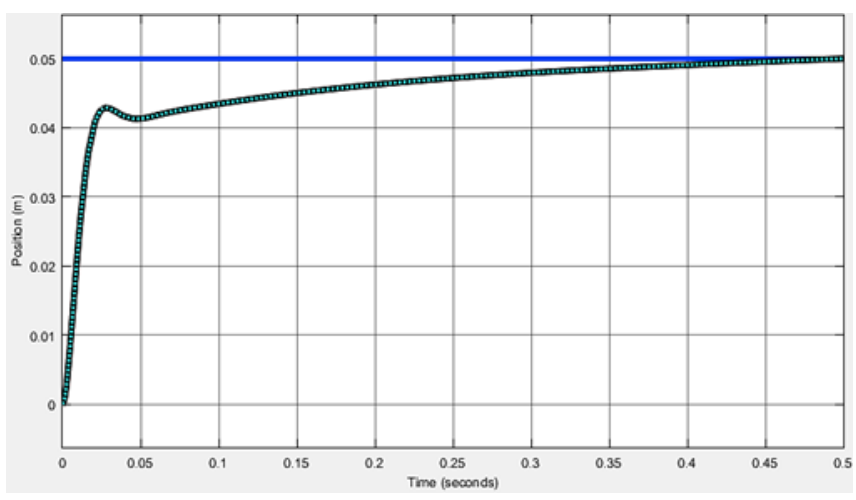

Fig. 7: PID controller's Step Response 
The above figure results for $\mathrm{K}_{\mathrm{p}}=120, \mathrm{~K}_{\mathrm{I}}=5$ $K_{D}=0.6$ and filter coefficient $N=100$, the pistons have settled in the Set value.

Increasing the gains values a steady state error occurs with values on the position of the pistons less than 0.05 m.

Increasing the Proportional gain while leaving the other two gains aside it is observed, that the system's response tries to follow the input set to it but a steady state error occurs. Increasing its value the steady state error is getting smaller but it is never eliminated.

Increasing the Integral gain and leaving the other coefficients intact a steady state error appears in the system with values of the position of the pistons greater than $0.05 \mathrm{~m}$.

By increasing the Derivative gain and leaving the other coefficients intact a steady state error appears in the system with values of the position of the pistons less than $0.05 \mathrm{~m}$.

\section{Results}

With the Proportional control method the Pneumatic system response gets accurate by choosing the correct value and acts fast with settling time $\mathrm{T} s=492.4 \mathrm{~ms}$ (Fig.4). When the application requirements are increased then this method of control gets unsuitable and the systems response with a load gets unpredictable [5]. Therefore this method of control cannot deal with all the scenarios that can occur in the industrial environment and needs furthermore assistance from a subsystem.

The second method of control that was used in the pneumatic system was the Proportional-Integral method which according to the theory using the integration term is intended to eliminate the steady state error in the system. The steady state error theoretically should have been eliminated by the integral term while adding some minor distortion on the systems response, has settling time $\mathrm{T} s=488.6 \mathrm{~ms}$ doesn't eliminate the stead state error Ess $=0.05 \mathrm{~m}$ as expected even at the maximum value (Fig 5).

In simulation environment the PI controller doesn't add oscillating behavior, nor creates overshoot in the pneumatic system. In reality studies have shown that the Integral coefficient on a Non-linear system adds constantly force in the system creating disturbances [58], and unbalancing the systems response. That is the main reason that the PI controller is avoided in general in both simulation experiments as well as in real once.

The next method of control that was applied in the pneumatic system was the PD controller (ProportionalDerivative). The-D coefficient should minimize the systems overshoot. With experimental testing it is confirmed that the PD controller helps stabilize the Pneumatic system response with $\mathrm{T} s=498.7 \mathrm{~ms}$ and eliminates the over shoot with a Ess=0.05 m (Fig.6).

The last controller tested was the three-term PID controller (Proportional-Integral-Derivative) to complete the comparison between all the controllers. The response time of the PID controller it's the same as the PD's. $\mathrm{T} s=498.5 \mathrm{~ms}$ (Fig.7). In simulation environment, PID controller has distortion when the coefficients value are too high but he is eliminating the overshoot effect.

In reality the presents of the I-term is creating low frequency signals that are hard to eliminate and adds unpredictable behavior in the system's response [5-8], making it hard to justify its usage on a non-linear model as this one.

Therefore the most optimal response for the six actuator pneumatic systems is given by PD controller in Simulation as well as in real experiments.

\section{Further upgrades}

By taking advantage of the possibilities given by Matlab/Simulink the pneumatic system that was designed for the purpose of finding the most optimal method of control, could be benefit by adding fuzzy logic controller. The particular controller is not found in the literature, but could be used to control the position of the six pistons of the system, providing new comparison data and therefore new results.

Fuzzy control occurred from the need for a control strategy that is not based on a mathematical model system, which usually differs from the real. The main structural elements of a fuzzy logic controller are the knowledge base, the fuzzy sets, the fuzzifier, the inference engine and the defuzzifier [9].

\section{Conclusion}

In the current study a comparison was performed between classic methods of control in simulation environment that included a six actuator robotic arm with six degrees of freedom and a single classic controller. The design and implementation of the pneumatic system was described (Matlab/Simulink).

The mathematical models of the directional valve, the actuators and the controllers were described. The experimental result where Proportional (P), ProportionalIntegral (PI), Proportional Derivative (PD), and Proportional-Integral-Derivative (PID) were used, were illustrated.

The innovative pneumatic system simulation model of an input and multiple output (Single Input, Multiple Output - SIMO), gives the user the ability to tune in a single controller that can simultaneously drives six parallel connected drive pistons.

After the experiments completion it was shown that the Proportional Derivative (PD) provided the system with the most optimal response.

Moreover, the specific pneumatic system with the given robustness and repeatability, as recorded by the experimental procedure, created a clear practical promises for the usage of the robotic hand ExoHand of Festo which can be used as an outer skeleton that can be worn as a glove by the human hand.

The fingers can be actively moved and their strength amplified the operator's hand movements are registered and transmitted to the robotic hand in real time.

The exoskeleton hand has all the principal physiological degrees of freedom of its human counterpart. It thus 
supports the human hand's diverse techniques for grasping and handling objects.

The objectives are to enhance the strength and endurance of the human hand, to extend humans' scope of action and to secure them an independent lifestyle even at an advanced age.

Since all the joints and their drive units are located outside the actual hand in the form of the exoskeleton, this manual orthosis can be fitted not only over the human hand, but also over an artificial handmade of silicone. Using the same hardware, this enables a scenario that creates a link between robotics and orthotics in a completely new way.

The ExoHand combines human intelligence with the capabilities of a robot. While machines are precise, robust and powerful, their responses to complex situations are limited. They normally rely on the visual and tactile perception and decision-making capacity of a human operator.

With the ExoHand the operator has the sensation of feeling the shape of the remote object. The human sense of touch can thus be implemented over long distances and can even be applied at the interface of the real to the virtual world. The system amplifies the strength of the human hand and helps employees remain in the work process for a longer time without suffering permanent physical effects. To prevent fatigue, the ExoHand can be worn for activities carried out in the assembly process, thereby functioning as an assistance system that makes for more pleasant working conditions in the assembly environment.

The ExoHand moving by eight pneumatic actuators which ensure the precise movement of the robotic arm. The forces, the angles and distances recorded by sensors [10].

Given the fact that the construction is fully operational, but there is no literature to be found with some effort to make simulations in the MatLab Simulink, neither any experimental results of its usage. The model of this paper performs robust control and can simulate human movement using six pneumatic actuators, which in fact will ensure the accurate movement of the robotic hand in real time.

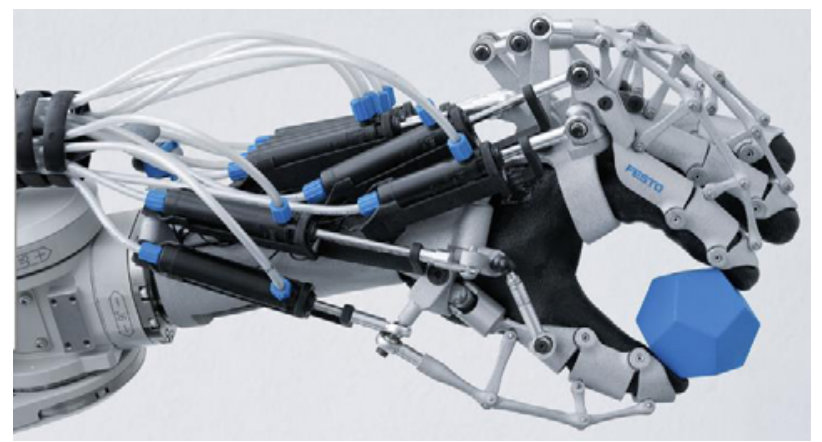

Fig. 8: Festo ExoHand
All authors would like to express their gratitude to the Piraeus University of Applied Sciences for providing the funding in order to undertake and complete this research project as part of: "Automation of Production and Services" Post graduate program of studies.

\section{References}

[1] Hydraulic-Pneumatic systems and Applications, Athanasios T. Routoulas, Contemporary Publications, Athens, Greece (2008)

[2] Movement control in the pneumatic systems, N. Themelis, Department of Mechanical Engineering, National Technical University of Athens, Greece (2007)

[3] Automation and Automatic Control Systems, Part A, I. Lignos, P. Bouslis, G. Politis, G. Chamilothoris, Electrical Sector, Athens (2001)

[4] A High Performance Pneumatic Force Actuator System Part 1 - Nonlinear Mathematical Model, Edmond Richer and Yildirim Hurmuzlu, Southern Methodist University, School of Engineering and Applied Science, Mechanical Engineering Department, Dallas (2001)

[5] "A comparative Investigation Into ProportionalIntegral-Derivative Control Methods for Accurate Position Control of Linear Pneumatic Actuators", M.G. Papoutsidakis, University of the West of England, Bristol, UK (2004)

[6] An Analysis of a Pneumatic Servo System and Its Applications to a Computer-Controlled Robot, S. Liu and J. E. Bobrow, Journal of Dynamic Systems, Measurement and Control, vol 110, PP 228-235 (1988)

[7] Non-conventional adaptive control of a servopneumatic unit for vertical load positioning, C. Ferraresi, P. Giraudo, G. Quaglia, West Technical Conference (1994)

[8] Variable Structure Control of a Pneumatic Actuator, Tang J. and Walker G., Transactions of the ASME, vol.117, pp.88-92 (1995)

[9] Control wind turbine induction motor squirrel cage using fuzzy logic, C. Kokkotas, Department of Electrical and Computer Engineering, University of Patras, Greece (2013)

[10] https://www.festo.com/net/SupportPortal/Files/1567 34/Brosch_FC_ExoHand_EN_lo.pdf 